RICE UNIVERSITY

\title{
A Terahertz Two-wire Waveguide with Low Bending Loss
}

\author{
by \\ Marx Mbonye \\ A THESIS SUBMITTED \\ IN PARTIAL FULFILLMENT OF THE \\ REQUIREMENTS FOR THE DEGREE \\ Master of Science
}

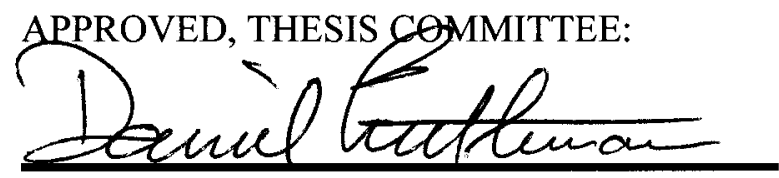

Daniel Mittleman, Professor, Chair

Electrical and Computer Engineering

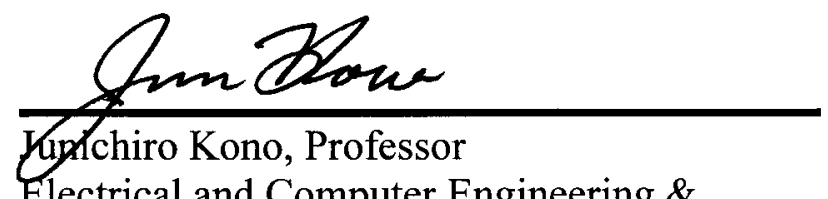

Electrical and Computer Engineering \&

Physigs and Astronomy

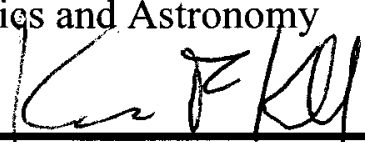

Kevin Kelly, Associate Professor

Electrical and Computer Engineering

HOUSTON, TEXAS

September 2009 
UMI Number: 1486036

All rights reserved

INFORMATION TO ALL USERS

The quality of this reproduction is dependent upon the quality of the copy submitted.

In the unlikely event that the author did not send a complete manuscript and there are missing pages, these will be noted. Also, if material had to be removed, a note will indicate the deletion.

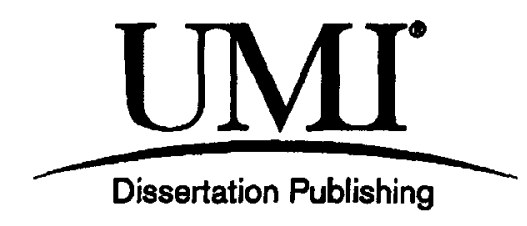

UMI 1486036

Copyright 2010 by ProQuest LLC.

All rights reserved. This edition of the work is protected against unauthorized copying under Title 17, United States Code.

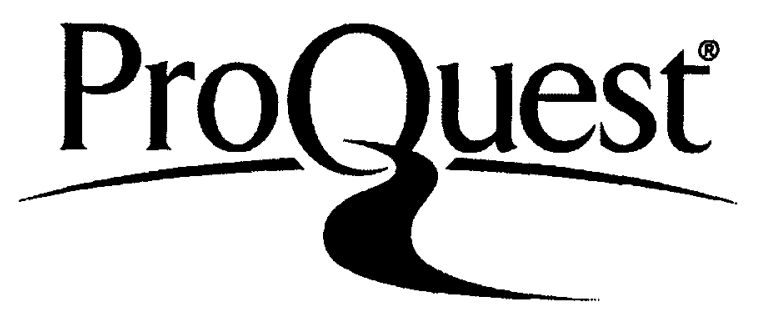

ProQuest LLC

789 East Eisenhower Parkway

P.O. Box 1346

Ann Arbor, Ml 48106-1346 


\section{ABSTRACT}

A Terahertz Two-wire Waveguide with Low Bending Loss

by

Marx Mbonye

We present experimental and theoretical evidence for low loss terahertz propagation along a two-wire waveguide. We find that the mode pattern at the end of the waveguide resembles that of a dipole consistent, with the expected fundamental mode of the structure. We experimentally observe that the two-wire configuration can be used to efficiently excite the radial mode (Somerfield wave) of a single wire, by adiabatically separating the two wires into two single wires. We observe for the first time that a commercial $300-\Omega$ TV twin-lead antenna cable can be used to propagate low loss $\mathrm{THz}$ radiation, guiding frequency components up to $0.2 \mathrm{THz}$ even though they are designed only to operate to about $800 \mathrm{MHz}$. We find this to be the case even at considerable wire-bend angles. 


\section{Acknowledgements}

I would like to thank my advisor, Dr. Daniel Mittleman, for his guidance and support. I would also like to thank Rajind Mendis for the same, more so for convincing Dan and I that it was worth testing the standard TV-twin lead antenna cable. I am grateful to Victoria Astley, William Chan and Joong-Wook Lee for valuable discussions and contributions to this work. Last I would like to acknowledge the strong support from my siblings Evelyne, Uri, Robert and Frantz and my loving parents Dr. Manasse Mbonye and Mrs. Joy Mbonye. 


\section{Contents}

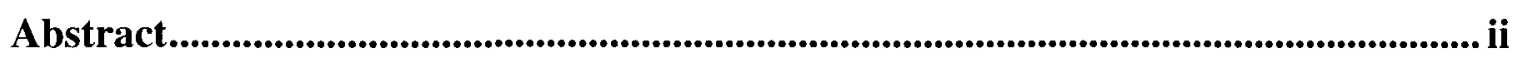
Acknowledgements ..............................................................................................................iii

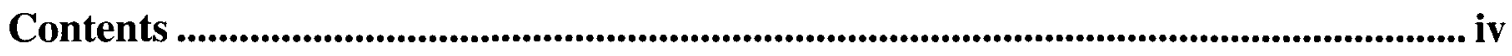

1. INTRODUCTION AND PRELIMINARY WORK................................................... 1

2. MEASUREMENTS WITH BARE METAL WIRES........................................................ 7 3. MEASUREMENTS WITH A COMMERCIAL 300- $\Omega$ TV ANTENNA CABLE ..............................................................................................................................................17

4. CONCLUSIONS ..................................................................................................................... 20

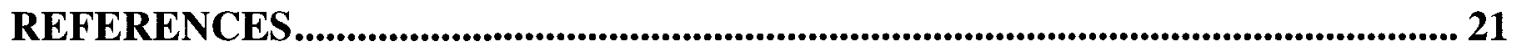




\section{INTRODUCTION}

Over the past decade, tremendous progress has been made in the study of terahertz $(\mathrm{THz})$ waveguides. Single mode coupling and propagation of $\mathrm{THz}$ radiation has been demonstrated in circular and rectangular metal waveguides, ${ }^{1}$ dielectric fibers, ${ }^{2}$ plastic ribbons, ${ }^{3}$ parallel-plate waveguides, ${ }^{4-6}$ bare metal wires, ${ }^{7-10}$ hollow glass waveguides, ${ }^{11}$ planar plasmonic devices, ${ }^{12}$ photonic crystal fibers, ${ }^{13,14}$ and hollow polymer optical fibers. ${ }^{15}$ In particular, a bare metal wire has attracted a great deal of attention for several reasons, including the simplicity of the design and its connection to plasmonics. ${ }^{16}$ Nevertheless, since the primary mode (the Sommerfeld wave) of a single wire is radially polarized, the commonly used linearly polarized photoconductive antennas cannot be used for the efficient excitation of this mode, and thus efficient coupling to a single wire requires the use of novel photoconductive antennas. ${ }^{10}$ Moreover, the weakly guiding nature of the Sommerfeld wave leads to high losses when the wire is bent, which limits the practical applications of this waveguide. ${ }^{8,9}$

Not too long ago, we demonstrated ${ }^{17}$ through simulation and experiment that one could couple a linearly polarized surface wave from free space at a 45 degree angle to a two-wire waveguide. For the simulation work we employed a commercially available finite element modeling (FEM) software (COMSOL Multiphysics).

In the simulation geometry, each of the wires is $7 \mathrm{~cm}$ long and $0.6 \mathrm{~mm}$ in diameter. The wire separation is $1 \mathrm{~mm}$. A schematic of the simulation geometry is shown in Fig. 1.1. The long axis of the wires is in the z-direction. Another wire (also $0.6 \mathrm{~mm}$ in diameter) is placed between the two wires and aligned perpendicular to them to act as an input coupler. This scattering mechanism for coupling to the propagating mode of the 
waveguide is similar to the one used in earlier work in our laboratory on single wire waveguides. ${ }^{9}$ A cylinder is placed in the model geometry such that its central axis lies at the center of the gap between the waveguide wires and the coupler wire. The cylinder is placed there in order to provide a location for the excitation source. The excitation plane is on the far circular face of the cylinder. The excitation is a linearly polarized ( $\mathrm{x}$ direction) wave with a frequency of $100 \mathrm{GHz}$ modeled as a plane wave with its k-vector incident on the gap between the wires and the input coupler at an incident angle of 45 degrees. This polarization is chosen such that the vector of the incident electric field is normal to the length axis of the two wires as illustrated in Fig. 1.1.

The outer boundary of the two wires is assigned a transition boundary condition with surface impedance defined by the conductivity of the metal and its skin depth. ${ }^{18}$ The simulation is bounded by enclosing the waveguide geometry in a rectangular box of air $(9 \mathrm{~cm} \times 2.5 \mathrm{~cm} \times 2 \mathrm{~cm})$, the walls of which are assigned a low-reflecting boundary condition to minimize the effects of back reflections. Before solving, the model is divided into 0.8 million tetrahedral mesh elements resulting in a model containing 1.1 million degrees of freedom. The model problem is then solved using an iterative Generalized Minimal Residual iterative Solver (GMRES) with Symmetric Successive Overrelaxion (SSOR) matrix preconditioning. ${ }^{19}$ Fig. 1.2(a) below shows result of the simulation with the wires present, whereas Fig. 1.2(b) shows the result with the wires absent. 


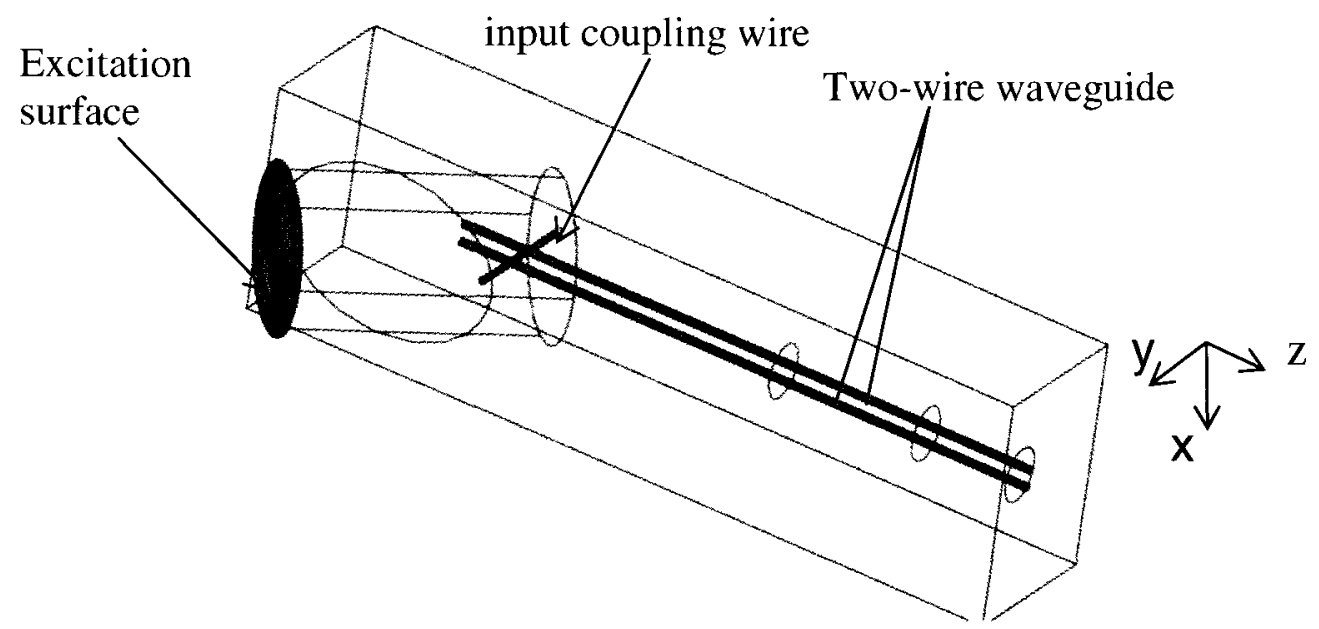

Fig.1.1. A 3D drawing of our simulation model geometry.
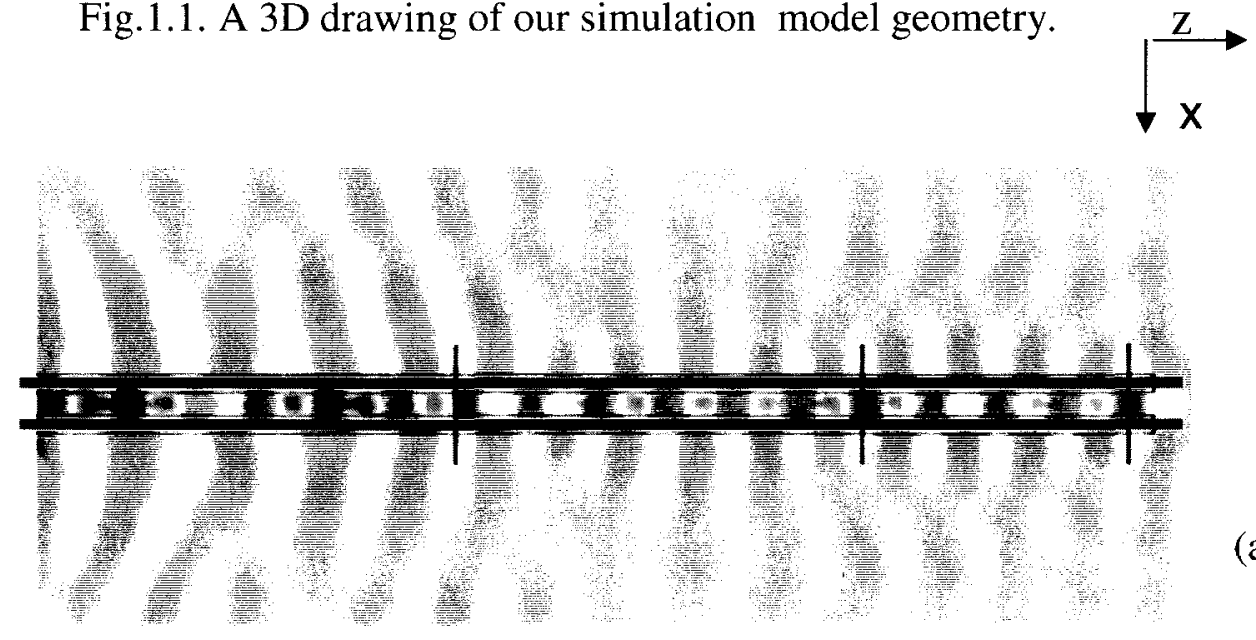

(a)

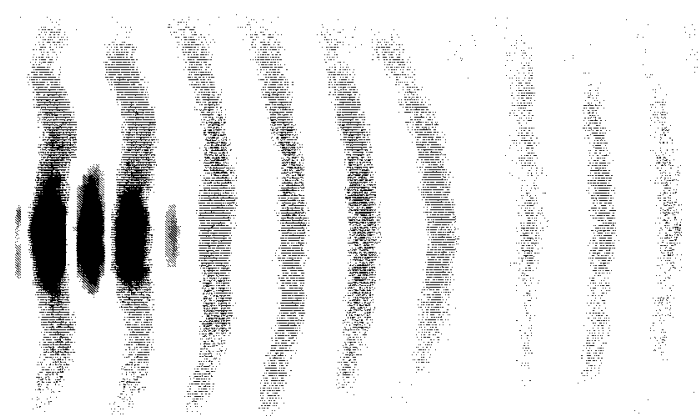

(b)

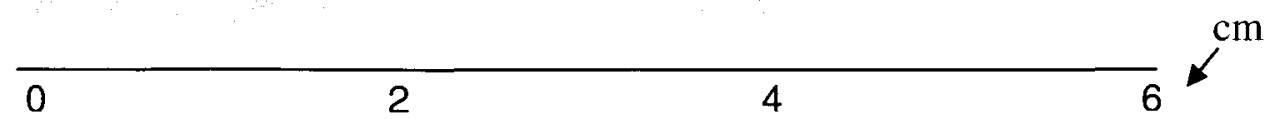

Fig. 1.2. (a). A slice plot of the electric field (x-component) in the $x-z$ plane showing the wave propagating in the positive $\mathrm{z}$ direction with the wires present. The wires are shown in bold. (b). A slice plot of the electric field (x-component) in the $x-z$ plane showing the wave propagating in the positive $\mathrm{z}$ direction with the wires absent. 
Fig. 1.2 (a) shows a slice plot of the electric field (x-component) in the $\mathrm{x}-\mathrm{z}$ plane of the wave propagating between the two wires. Red indicates a large positive electric field, whereas blue indicates a large negative electric field. There is clear evidence of a guided mode propagating along the two wires, with the largest fields concentrated in the region between them. One can clearly distinguish coupling in the domain between the wires and radiation outside the domain, by noting that the waves between the wires and those outside the wires are out of phase. Fig. 1.2 (b) shows a slice plot of the electric field (x-component) in the $\mathrm{x}-\mathrm{z}$ plane of the wave propagating with the wires absent. It is apparent that the guided mode is absent without the wires.

A similar geometry as in the simulation is used on our experiment. A schematic of the experimental set-up is shown in Fig. 1.3. It consists of a terahertz transmitter/receiver pair (fiber coupled photo-conductive antennas) and two cylindrical stainless steel metal wires. Each of the wires is $7 \mathrm{~cm}$ long and $0.6 \mathrm{~mm}$ in diameter. The wire separation is $2 \mathrm{~mm}$.

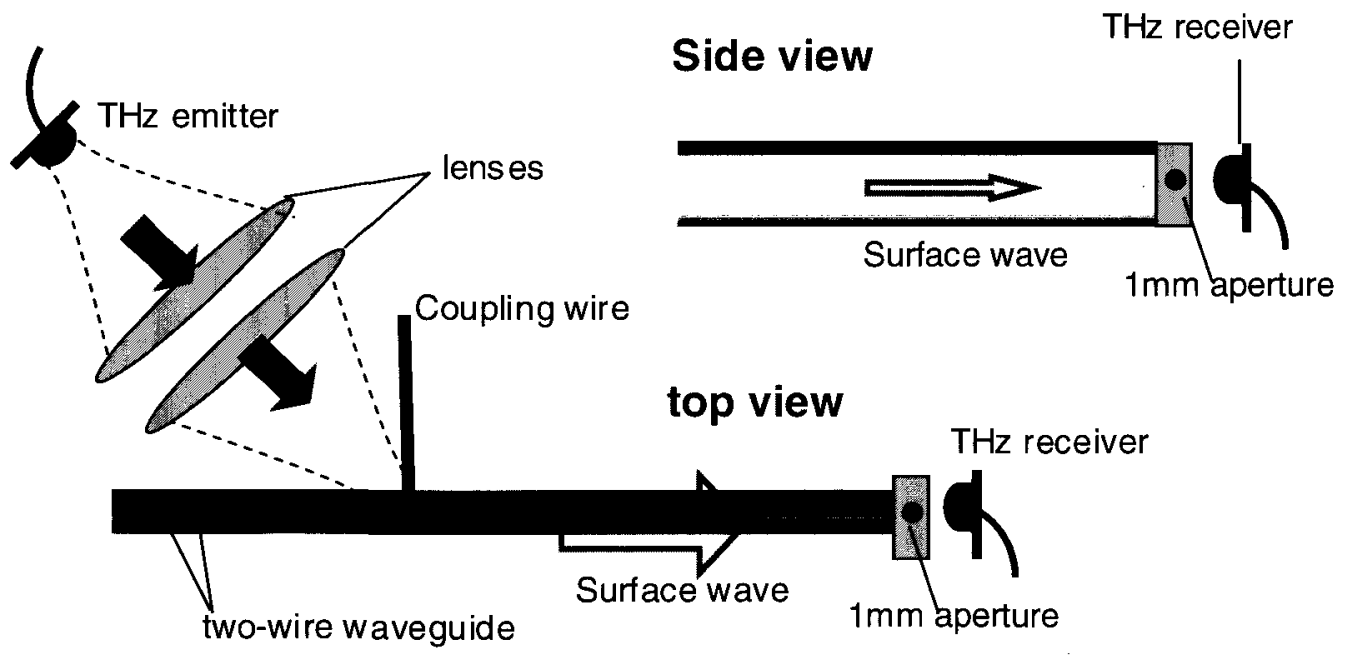

Fig. 1.3. A schematic showing our experimental set-up, whose geometry resembles that of the simulation 
The two-wire waveguide is supported using several pieces of Styrofoam nearly transparent to $\mathrm{THz}$ radiation. We isolate a $2 \mathrm{~mm}$ by $1.5 \mathrm{~mm}$ grid transverse-grid at the output end of the two wires. The grid has a total number of 63 points and the $\mathrm{THz}$ receiver is raster scanned along all these points in steps of $0.25 \mathrm{~mm}$. The field mapping is done for both the vertical transverse electromagnetic (TEM) polarization and for the horizontal polarization of the $\mathrm{THz}$ receiver, while keeping the polarization of the $\mathrm{THz}$ emitter undisturbed. Once the field mapping for both polarizations is done, both data sets are combined to generate the resultant electric field in magnitude and direction shown in Fig.1.4 (a). Fig. 1.4 (b) shows the corresponding simulation result for the mode pattern at the end of the two-waveguide with a wire separation of $1 \mathrm{~mm}$. Both figures indicate that the mode at the output end of the wires strongly resembles a dipole.

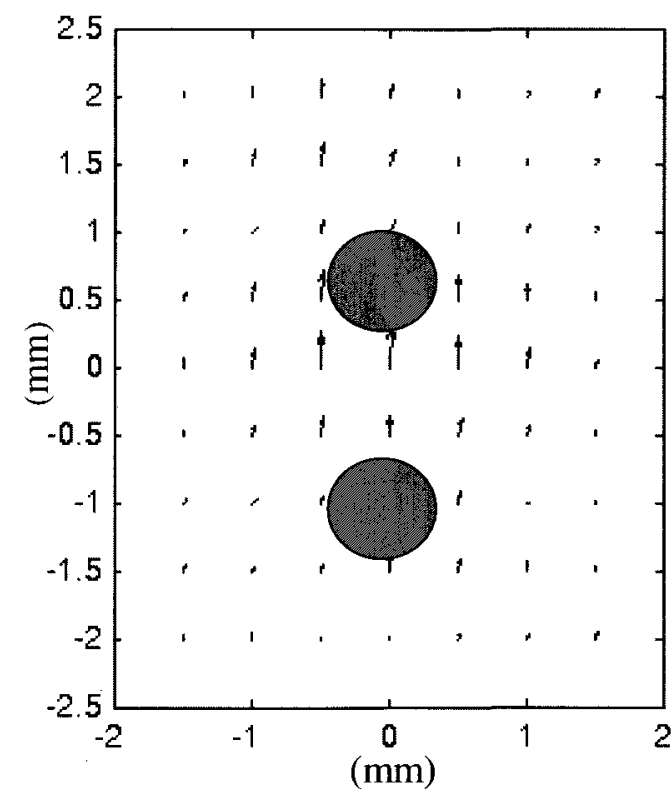

Fig. 1.4 (a). The experimental result showing the mode pattern at the output end of a two-wire waveguide

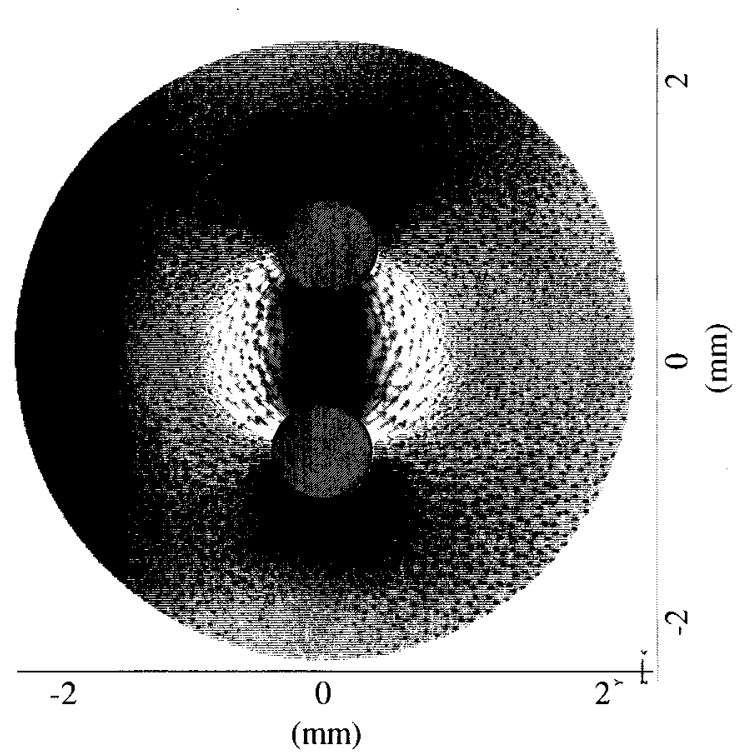

Fig. 1.4 (b). The corresponding simulation result showing the mode pattern at the output end of a two-wire waveguide 
In summary, preliminary simulation and experimental work shows strong evidence of wave-guiding and that the mode pattern at the end of the waveguide strongly resembles a dipole. In spite of these good results, the coupling of the wave, from freespace to the two-wire waveguide is relatively inefficient since the incoming wave is angled at $45^{\circ}$ and only a small percentage couples to the dipole mode of the waveguide.

In the next section of this thesis, we show that a linearly-polarized wave can be efficiently coupled in an end-fire configuration to one end of a two-wire waveguide, if the input polarization is parallel to a line connecting the wire centers (center-to-center line). We establish the excitation of the transverse electromagnetic (TEM) mode of this two-conductor structure by mapping the field pattern at the output, and compare the attenuation to predicted values. Significantly, we find that the bending loss is considerably lower than for an equivalent bend with a single wire waveguide. 


\section{MEASUREMENT WITH BARE METAL WIRES}

Our experimental setup consists of a $\mathrm{THz}$ transmitter/receiver pair (fiber coupled photoconductive antennas) as shown in the inset in Fig. 2.1. Two cylindrical stainlesssteel wires form the waveguide. They each have a diameter of $0.3 \mathrm{~mm}$ and a length of 24 $\mathrm{cm}$. The center-to-center wire separation is $0.5 \mathrm{~cm}$. The two-wire waveguide is supported using several pieces of Styrofoam which are nearly transparent to $\mathrm{THz}$ radiation. The waveguide is bent to an angle of about $45^{\circ}$ at the transmitter-end in order to distinguish between the guided mode and the (uncoupled) wave propagating freely in space. The THz waveform represented by the dashed curve in Fig. 2.1 is obtained when the two-wire waveguide is in place. The solid flat line is obtained when the waveguide is absent, with the $\mathrm{THz}$ emitter and receiver undisturbed, which indicates that there is no line-of-sight transmission of freely propagating radiation from the emitter to the receiver.

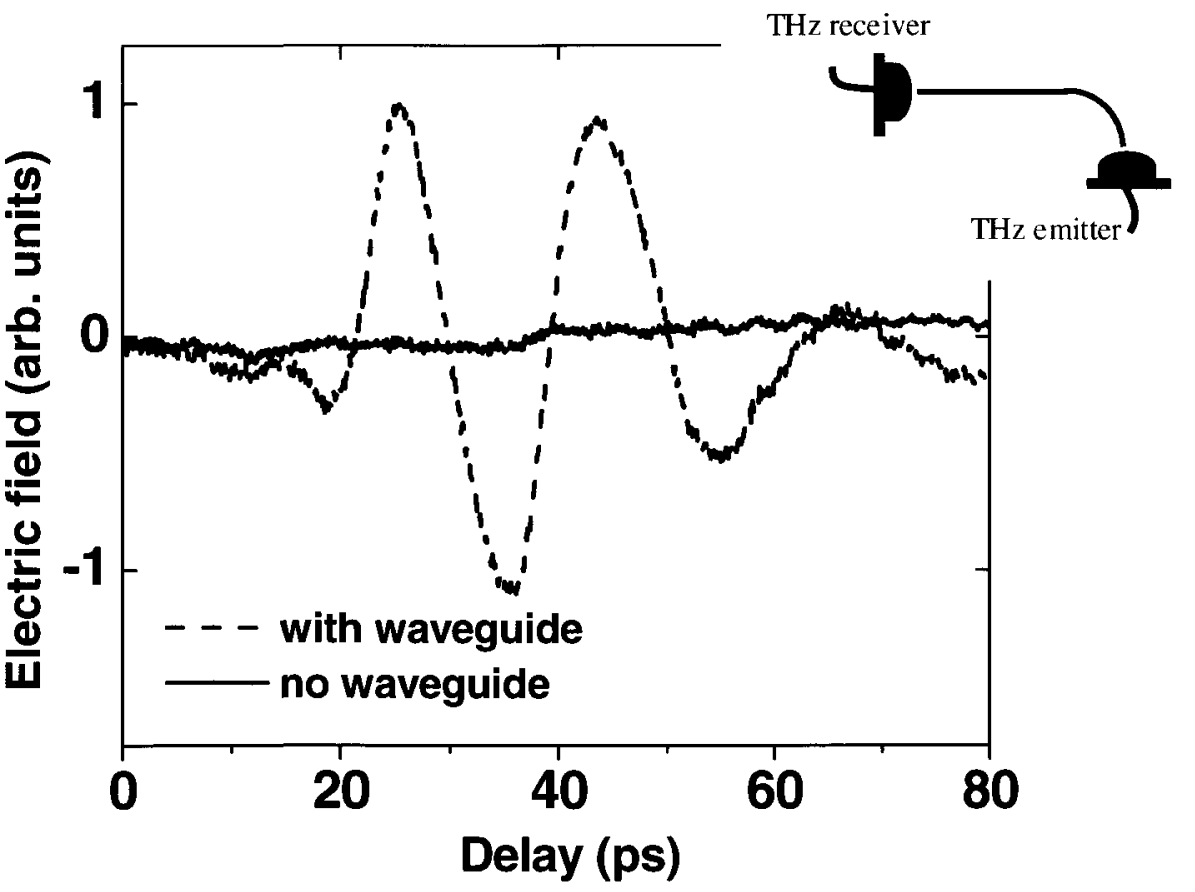

Fig. 2.1. Dotted line: Transmitted $\mathrm{THz}$ pulse along a $24 \mathrm{~cm}$ two-wire waveguide, $0.5 \mathrm{~cm}$ wire separation. Solid line: measured when the two-wire waveguide is absent with the $\mathrm{THz}$ emitter and receiver remaining fixed in position. The inset shows the schematic of our experimental set-up 
In the next experiment, we image the propagated electric field at the output end of the two-wire waveguide, in a plane perpendicular to the wire axes. The $\mathrm{THz}$ receiver (containing a $1.5 \mathrm{~mm}$ aperture for improved spatial resolution) is raster-scanned in a 8 $\mathrm{mm}$ by $6 \mathrm{~mm}$ grid in steps of $1 \mathrm{~mm}$. For the field mapping, both orthogonal electric field components of the propagating transverse-electromagnetic (TEM) mode were obtained, by orienting the receiver parallel and perpendicular to the center-to-center line. Once the two orthogonal components were measured, these data sets were combined to generate the total electric field in magnitude and direction. This result is shown in Fig. 2.2.

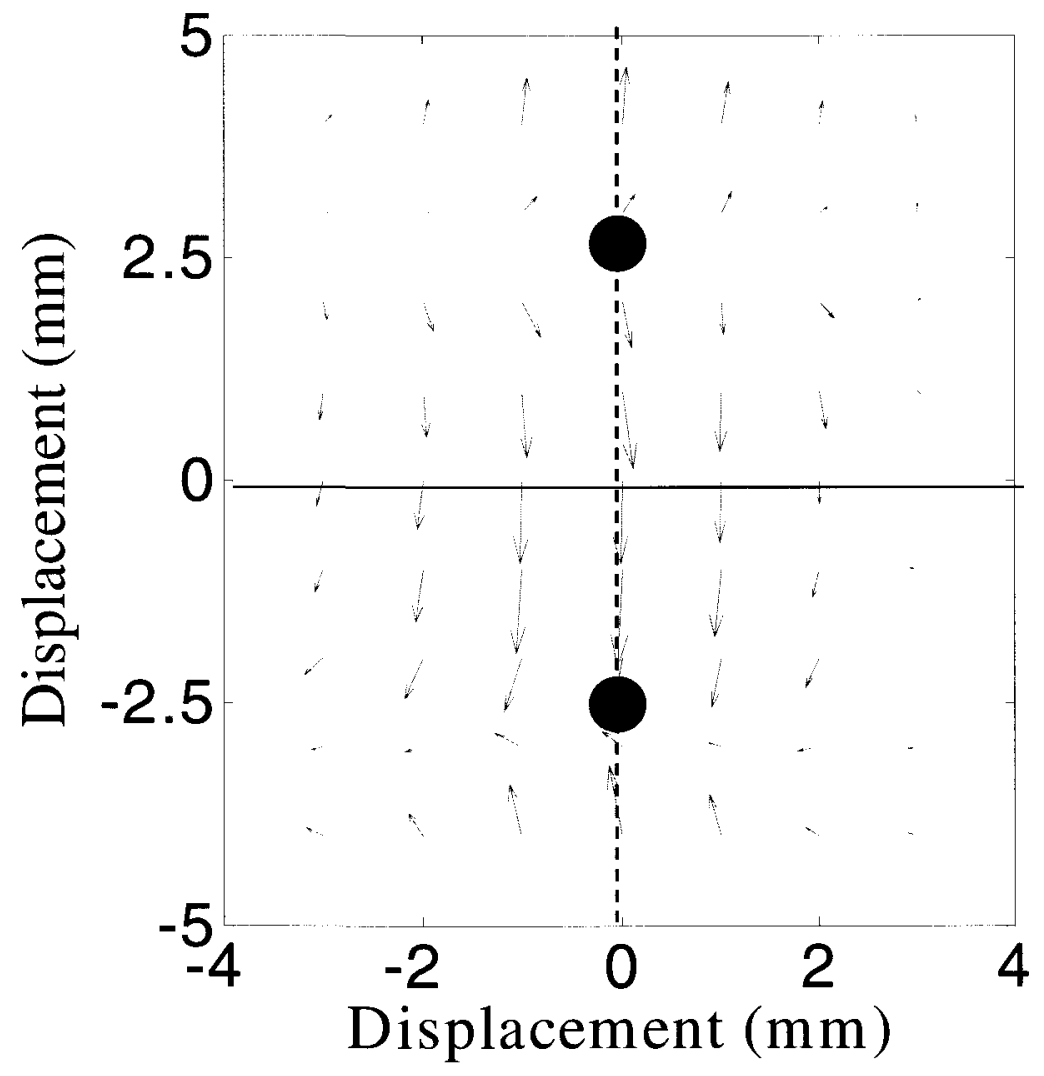

Fig. 2.2. The $\mathrm{THz}$ receiver is raster scanned around a $9 \mathrm{~mm} X 7 \mathrm{~mm}$ area at the output end of the waveguide. The field mapping is done for both the vertical (TEM) polarization and for the horizontal polarization of the $\mathrm{THz}$ receiver while keeping the polarization of the emitter undisturbed. The data sets for both polarizations are combined to generate the resultant electric field magnitude and direction shown in arrows. 


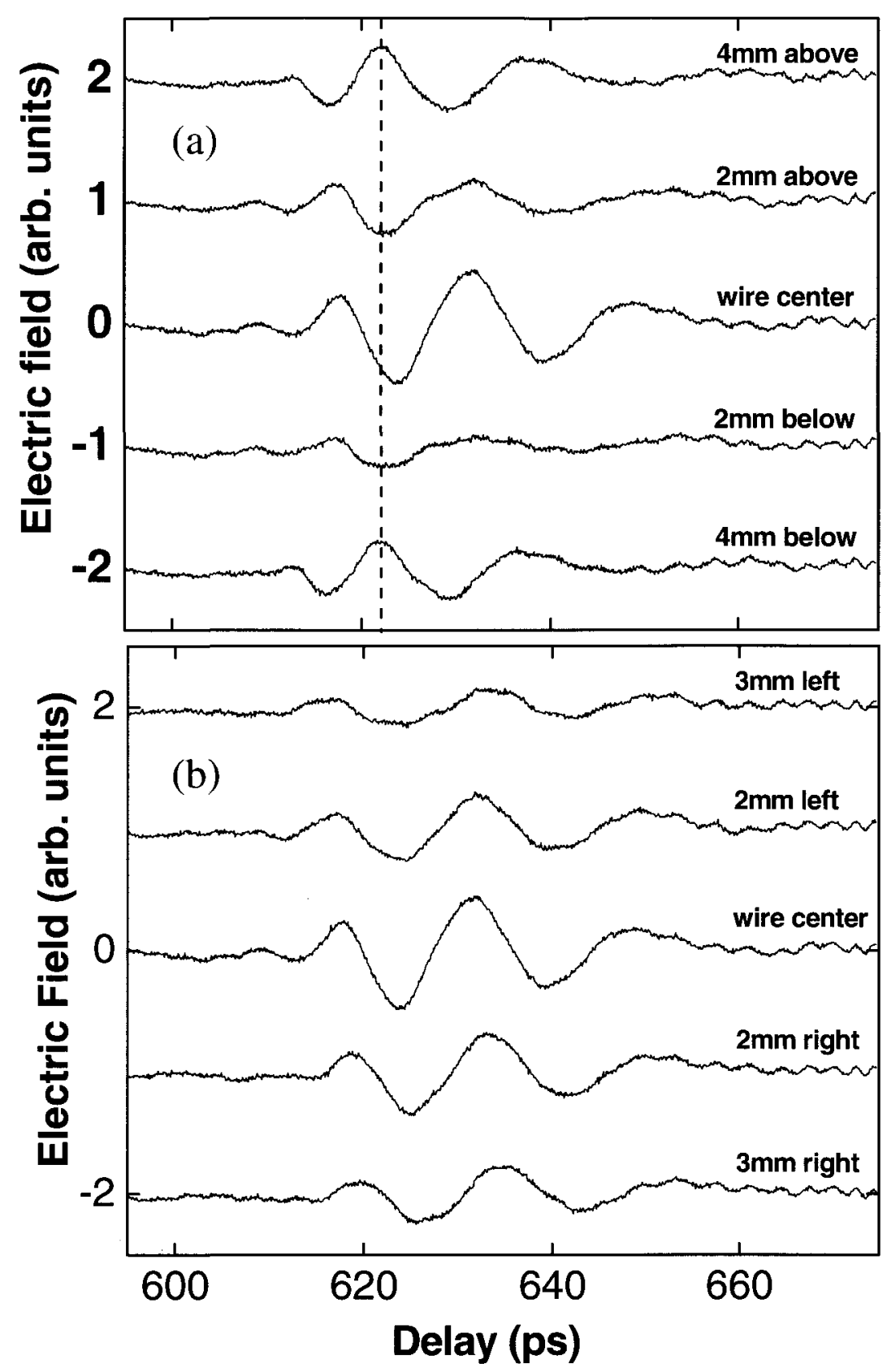

Fig. 2.3(a). THz waveforms for the points along the vertical dotted line in Fig 2.2. (b) $\mathrm{THz}$ waveforms for the points along the solid horizontal line in Fig 2.2. The waveforms have been shifted for clarity.

The strongest electric field is concentrated between the wires, which decreases as one moves away from that central area. Fig. 2.3 (a) shows the $\mathrm{THz}$ waveforms for points along the center-to-center line of the two wires in Fig. 2.2. The electric field amplitude is 
seen to decrease as one moves away from the center of the wires. In the signals corresponding to the top of the upper wire ( $4 \mathrm{~mm}$ above the waveguide axis), and bottom of the lower wire ( $4 \mathrm{~mm}$ below the axis), we observe a flip in polarity (relative to the field between the wires) due to the dipolar nature of the propagating TEM mode. Fig 2.3 (b) shows the $\mathrm{THz}$ waveforms for the points along the solid horizontal line in Fig 2.2. As anticipated the electric field signal decreases as one moves either left or right of the twowire center.

Next we determine the loss characteristics of the waveguide. The attenuation is measured by comparing $\mathrm{THz}$ pulses that have propagated through two different waveguide lengths, as in previous experiments. ${ }^{1-5,8,9}$ For both lengths, we use the same $45^{\circ}$ bend immediately after the transmitter, which introduces a fixed coupling and bending loss. We change only the length of the straight section, and extract the intrinsic loss on a straight waveguide by normalizing out these coupling and bending effects.

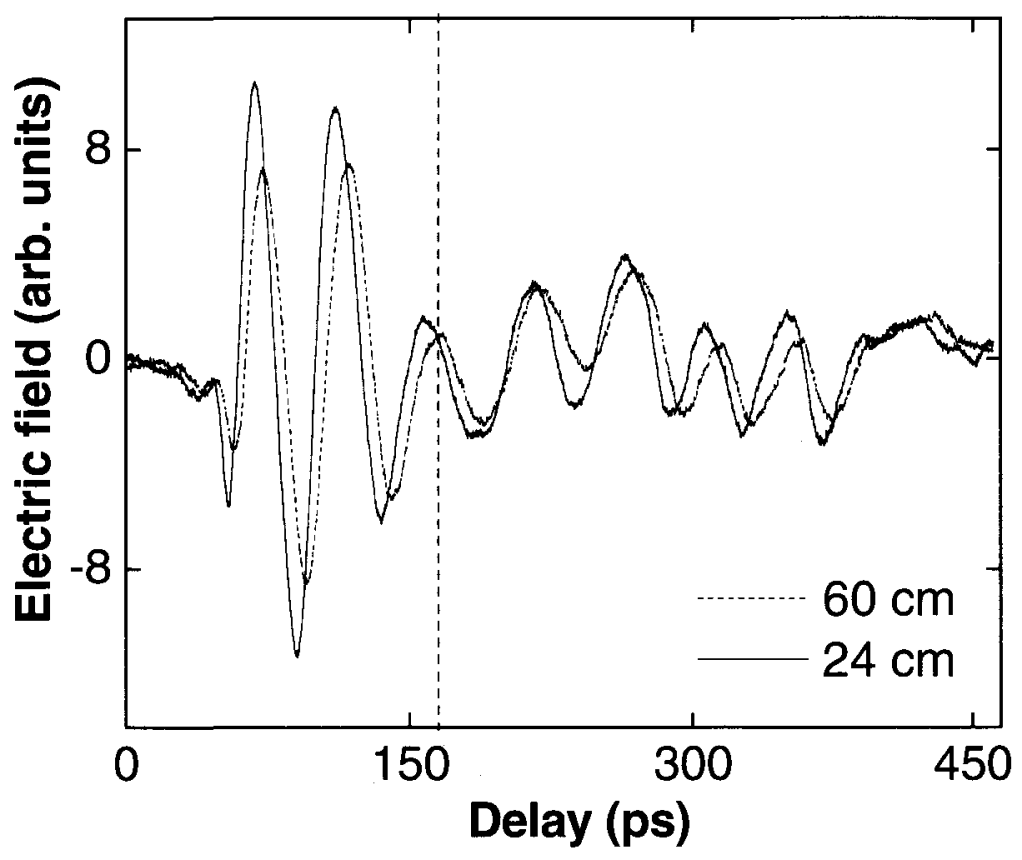

Fig. 2.4. Transmitted THz pulses along a $60 \mathrm{~cm}$ and $24 \mathrm{~cm}$ waveguide. 


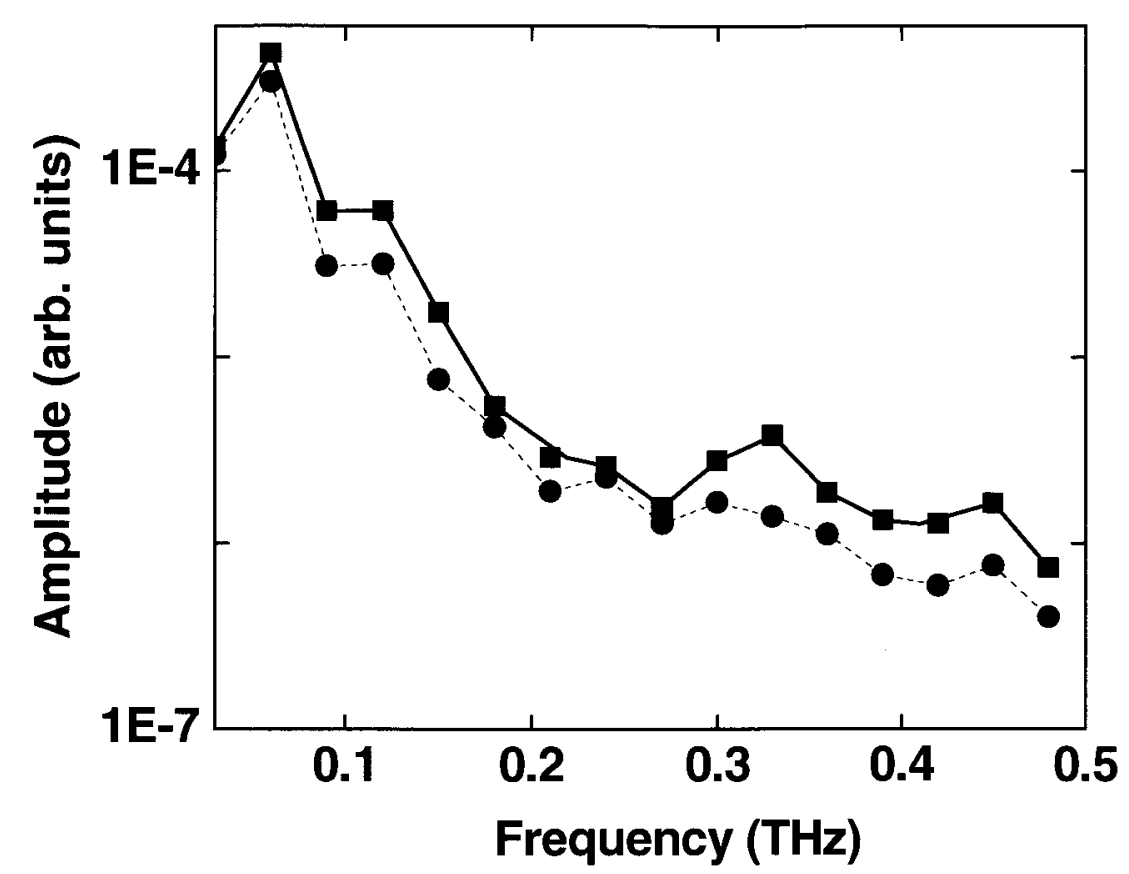

Fig. 2.5. Fourier transform spectra for the $\mathrm{THz}$ pulses truncated at the line shown in Fig.2.4.

The measured $\mathrm{THz}$ pulses from these two lengths are shown in Fig. 2.4. The two main pulses are almost identical in shape, indicating the negligible group velocity dispersion expected for a TEM mode. The $\mathrm{THz}$ pulses have a relatively long time scales of about 100 ps. The long time scales are due to the poor coupling efficiency of the input Gaussian beam to the two-wire waveguide. At high frequencies, the energy is more concentrated near the wire boundaries, and will be highly mis-matched with the input Gaussian profile, thus, part of the high frequencies will not be coupled into the waveguide. The relatively small change in amplitude signifies the low-loss nature of the propagation. The corresponding amplitude spectra obtained by Fourier transforming the truncated pulses (at the dashed line) are shown in Fig. 2.5.

The amplitude attenuation coefficient derived using the measured spectra is shown in Fig. 2.6. For comparison, the theoretical attenuation for the two-wire waveguide 
(TEM mode) ${ }^{20}$ and for a single-wire waveguide (Sommerfeld wave) are also shown. The cylindrical wires in these calculations were modeled as having a diameter of $0.3 \mathrm{~mm}$ and being made of type 304 stainless steel, with a conductivity of $1.45 \times 10^{6} \mathrm{~S} / \mathrm{m}$, as in our experiments. For comparison, we show the attenuation for the TEM mode of a stainless steel parallel-plate wave guide (PPWG) with a plate separation of $0.5 \mathrm{~cm}$. Based on Fig. 2.6, the measured attenuation of the two-wire waveguide is relatively low and is in reasonable agreement with its theoretical value.

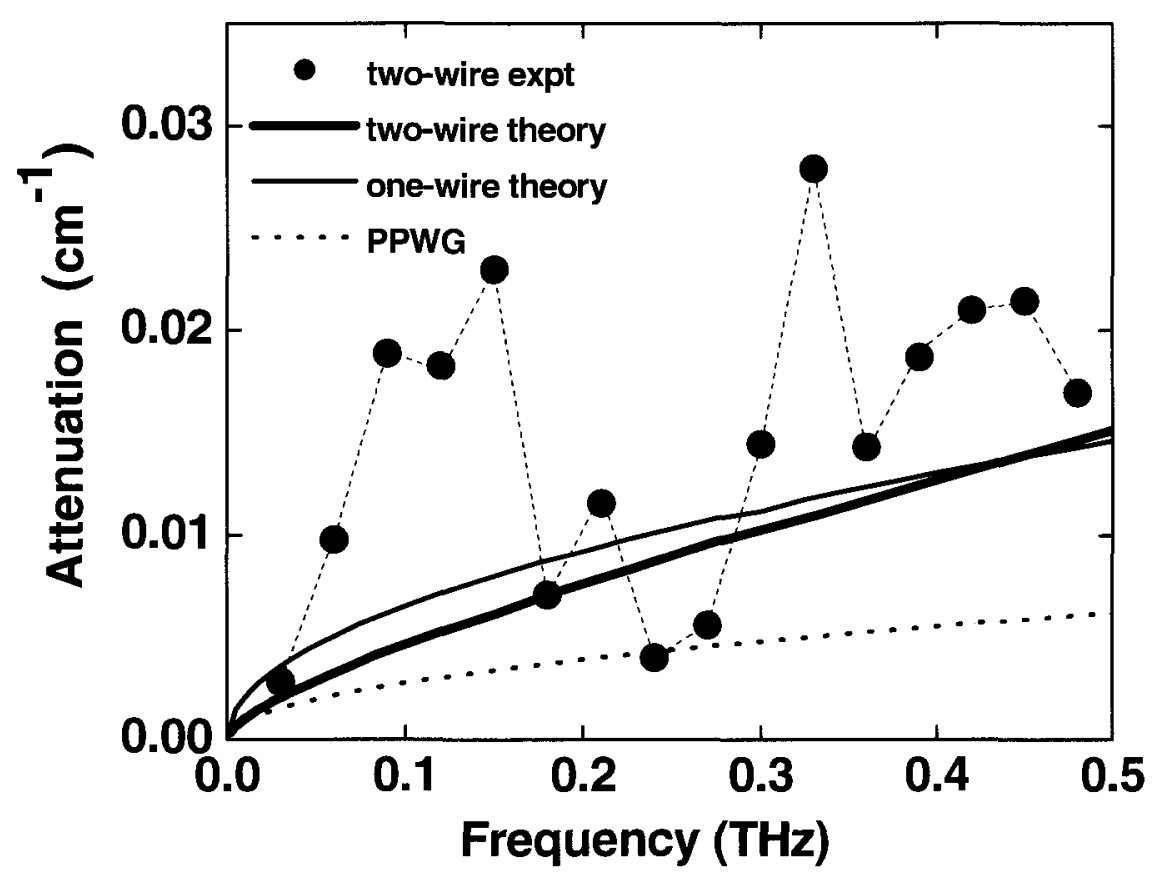

Fig. 2.6. Amplitude attenuation coefficient. Dots: measured for two-wire from $60 \mathrm{~cm}$ and $24 \mathrm{~cm}$ stainless steel, $0.5 \mathrm{~cm}$ wire separation. Thick curve: theory for two-wire for $0.31 \mathrm{~mm}$-diam stainless steel, $0.5 \mathrm{~cm}$ sep. Thin curve: Sommerfield theory for one-wire 0.31 - mm-diam stainless steel. Dotted curve: Theory for ideal parallel plate waveguide, $0.5 \mathrm{~cm}$ plate separation.

In addition to measuring the attenuation of the two-wire waveguide, we also measured the bending loss. Fig. 2.7(a) shows a schematic of the setup showing a $65 \mathrm{~cm}$ long waveguide. The straight section of the waveguide near the receiver-end is labeled 
$\mathrm{L} 1$, the bent section $\mathrm{S}$, and the straight section near the emitter-end L2. The bend angle is $\theta$ and the radius of curvature is $\mathrm{R}$. We keep the receiver and only vary the bend angle, and the location of the emitter (which is fixed relative to the straight section L1). Since the lengths L1, S, and L2 remained the same for every bend angle, any changes in transmission with changing $\theta$ result only from variations in the loss due to bending.

(a)
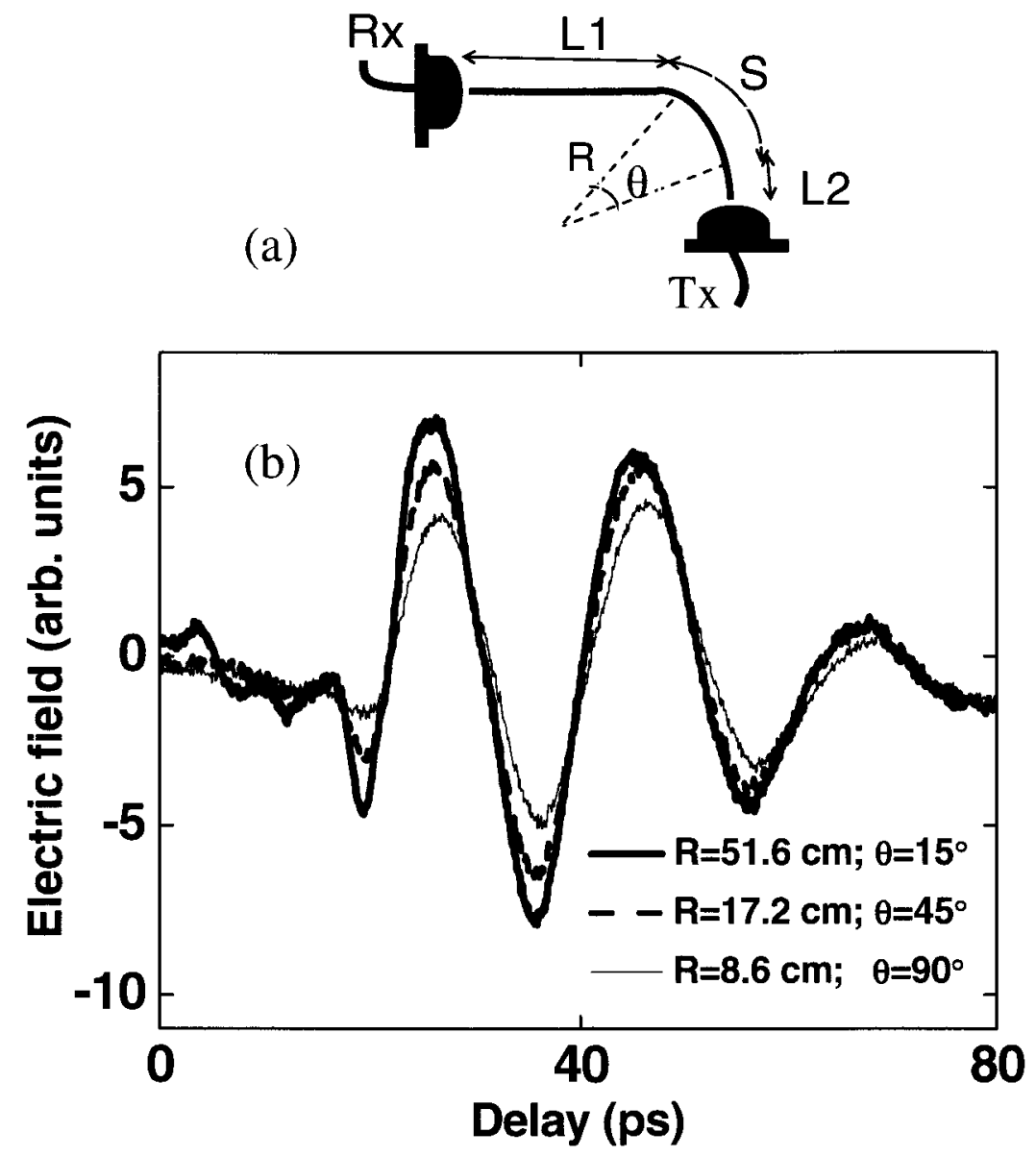

Fig. 2.7. (a). A schematic of the experimental set-up for the bending measurements. (b). Transmitted $\mathrm{THz}$ pulses along a $65 \mathrm{~cm}$ waveguide with various radii of curvature. Solid $\mathrm{R}=51.6 \mathrm{~cm}\left(\theta=15^{\circ}\right)$. Dashed line: $\mathrm{R}=17.2 \mathrm{~cm}$ $\left(\theta=45^{\circ}\right)$. Thin: $\mathrm{R}=8.6 \mathrm{~cm} \quad\left(\theta=90^{\circ}\right)$.

The time-domain waveforms for several values of R are shown in Fig 2.7 (b). As expected, the signal decreases with increasing bend angle, but the decrease is relatively 
slow. Even a relatively small radius $\left(\mathrm{R}=8.6 \mathrm{~cm}\right.$, corresponding to a $90^{\circ}$ bend) results in less than a factor of two change in the peak-to-peak $\mathrm{THz}$ field. This demonstrates the tight coupling of the propagating wave to the two wires, in contrast the Sommerfield wave of a single-wire where the wave is very weakly coupled. For an equivalent bend on a single wire waveguide ${ }^{9}$, the attenuation would be more than a factor of 10 , considerably larger than the value measured here which is less than a factor of two. Fig. 2.8 shows the peak-peak electric field, normalized to the value corresponding to no bend $\left(\theta=0^{\circ}\right.$, $R=$ inf).

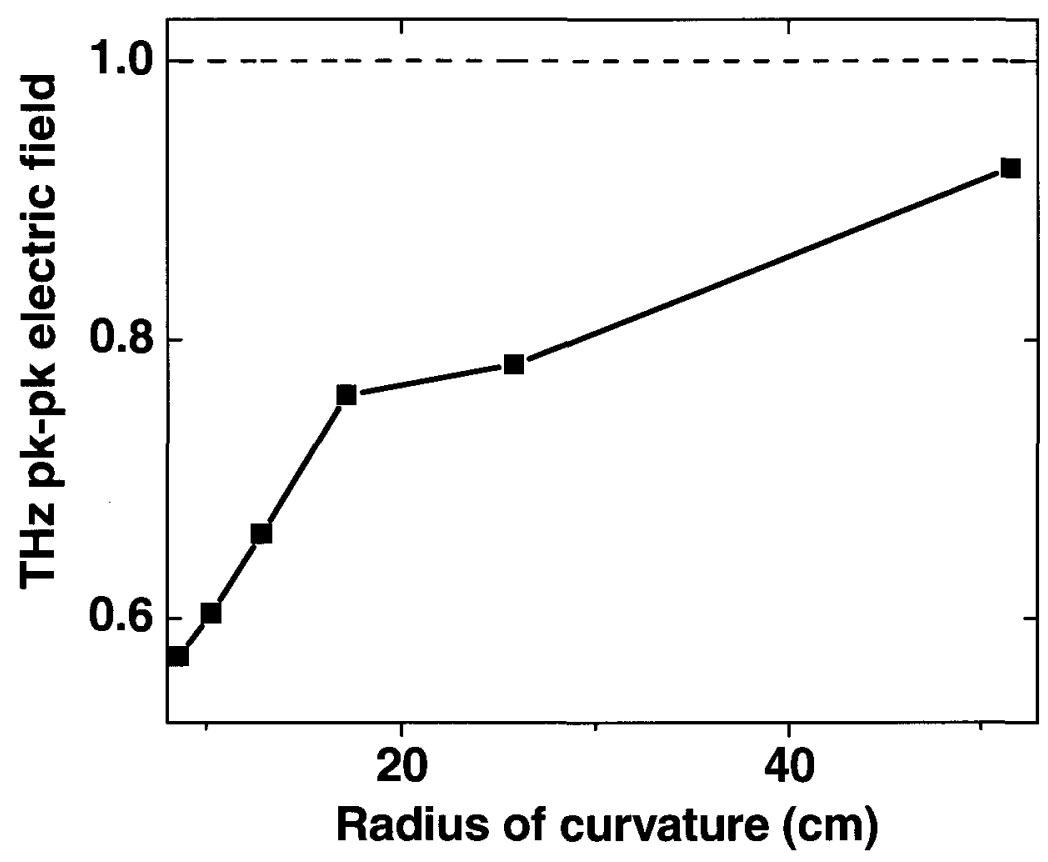

Fig. 2.8. The the peak-peak electric field, normalized to the value corresponding to no bend $\left(\theta=0^{\circ}, \mathrm{R}=\right.$ inf $)$.

In addition to measuring the ohmic and bending losses, we also computed and compared the losses for various wire separations. An experiment to quantify the influence of the wire separation on the loss would be very difficult to assemble because for each 
wire separation, the wire bend would have to be identical. Theoretical curves showing the losses for various wire separations are shown in Figure 2.9. It is apparent that the greater the wire separation the lower the conductor losses.

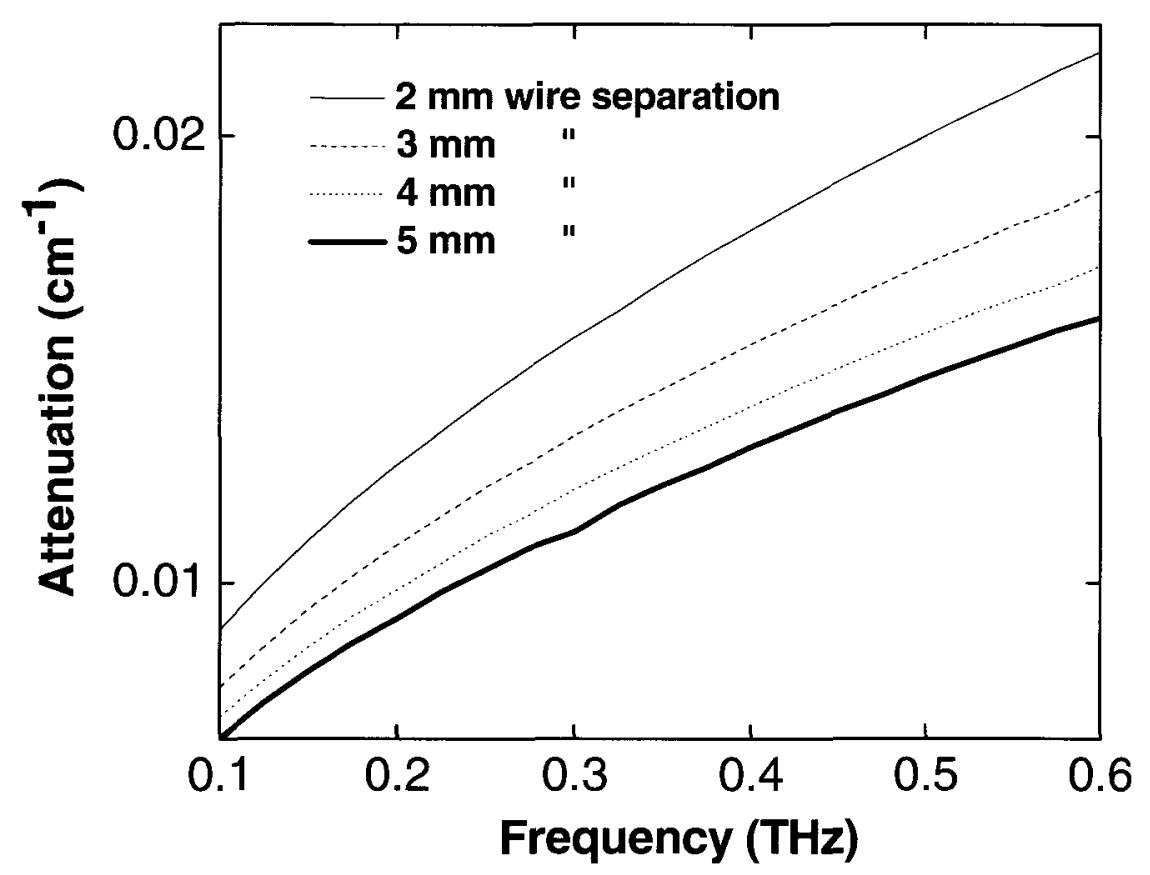

Fig. 2.9. Theoretical curves showing the influence of the wire separation on the conductor losses

We perform one more measurement with the bare metal wires whereby we slowly separate the two wires on the straight section close to the receiver. The receiver is moved $2 \mathrm{~mm}$ above the center of the straight wire as shown in Fig. 2.10, and the electric field is sampled. The receiver is then moved $2 \mathrm{~mm}$ below the center of the lower wire and the measurement is repeated. We find that the radial mode (Sommerfield wave) ${ }^{8,9}$ of a single wire is excited at the end of the straight wire and at the end of the separated wire. 


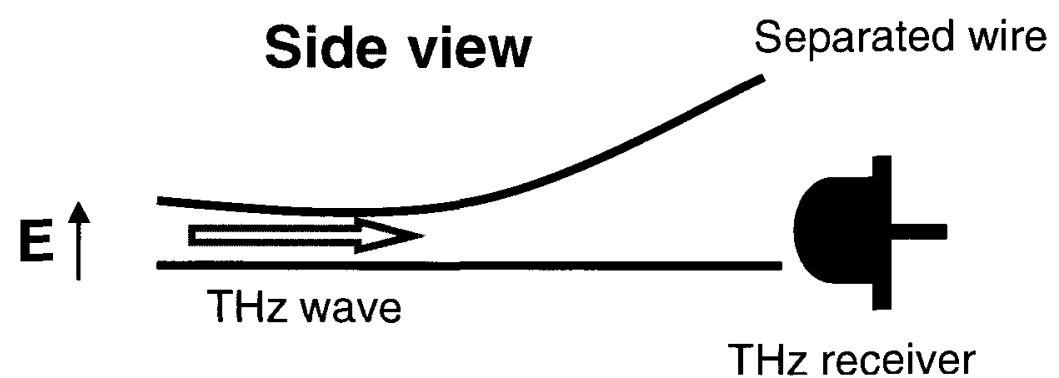

Fig. 2.10. A schematic of the experimental set-up showing how the wires are slowly separated close to the receiver

Fig. 2.11 shows measured time domain waveforms for the two-wire waveguide before separating the wires, and after separating them. Once the wires are separated, the $\mathrm{THz}$ electric field signal is sampled $2 \mathrm{~mm}$ above and $2 \mathrm{~mm}$ below the straight wire. The flip in polarity confirms that we are indeed looking at the radially polarized mode of a single wire. The same radially polarized field is observed in the separated wire when the electric field is sampled $2 \mathrm{~mm}$ above and $2 \mathrm{~mm}$ below it.

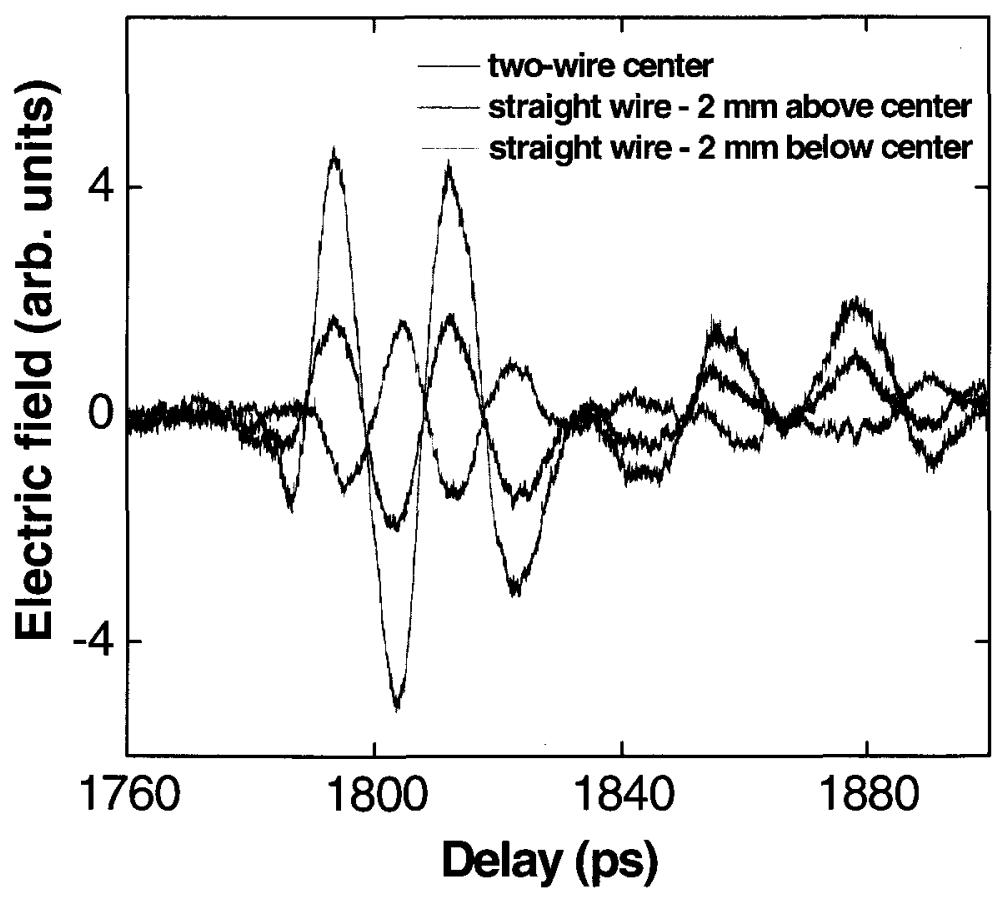

Fig. 2.11. Measured time domain waveforms for the two wire waveguide before separating the wires, and after separating them. The $\mathrm{THz}$ electric field signal is sampled $2 \mathrm{~mm}$ above and $2 \mathrm{~mm}$ below the straight wire. The flip in polarity is due to the radial nature of the field. 


\section{MEASUREMENTS WITH A COMMERCIAL 300- $\Omega$ TV-TWIN LEAD CABLE}

In addition to the work with bare metal wires, We also tested a commercially available $300-\Omega, 18$ gauge standard twin-pair TV antenna cable. This is basically a twowire waveguide, where the wires are equally spaced and completely enclosed within a dielectric medium (polyethylene), as shown in the inset of Fig. 3.1. Each wire consists of seven copper strands of about $500 \mu \mathrm{m}$ diameter woven together. The center-to-center separation is about $0.3 \mathrm{~cm}$.

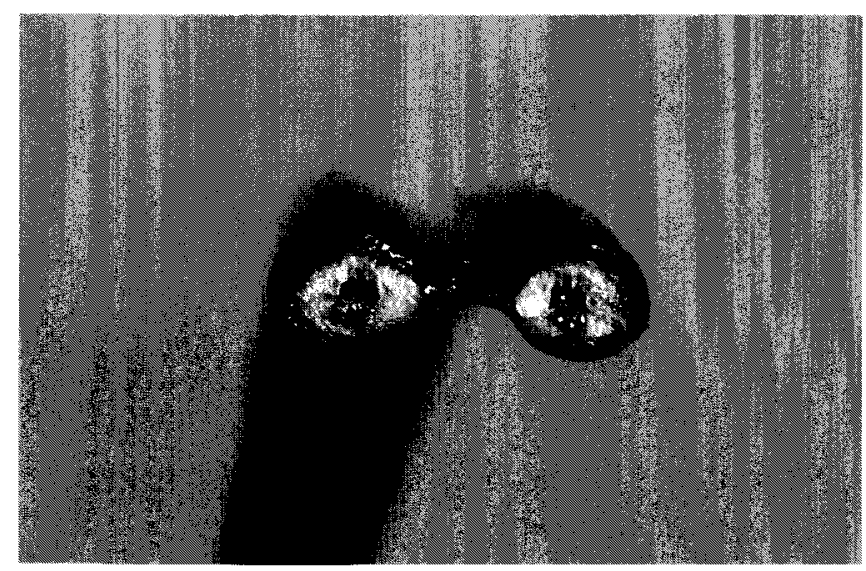

Fig. 3.1. A picture of the TV-antenna twin lead cable that we used.

The length of the cable used in our measurements is $9.5 \mathrm{~cm}$. We insert this length of cable between the $\mathrm{THz}$ transmitter and receiver, incorporating a $90^{\circ}$ bend so that no freely propagating radiation can be detected. After measuring the propagated signal, the antenna cable is removed, and the measurement is repeated with the emitter and receiver undisturbed. Finally, the receiver is re-positioned to face the emitter (with the distance between them kept at $9.5 \mathrm{~cm}$ ), and the measurement is repeated for the freely propagating beam. 


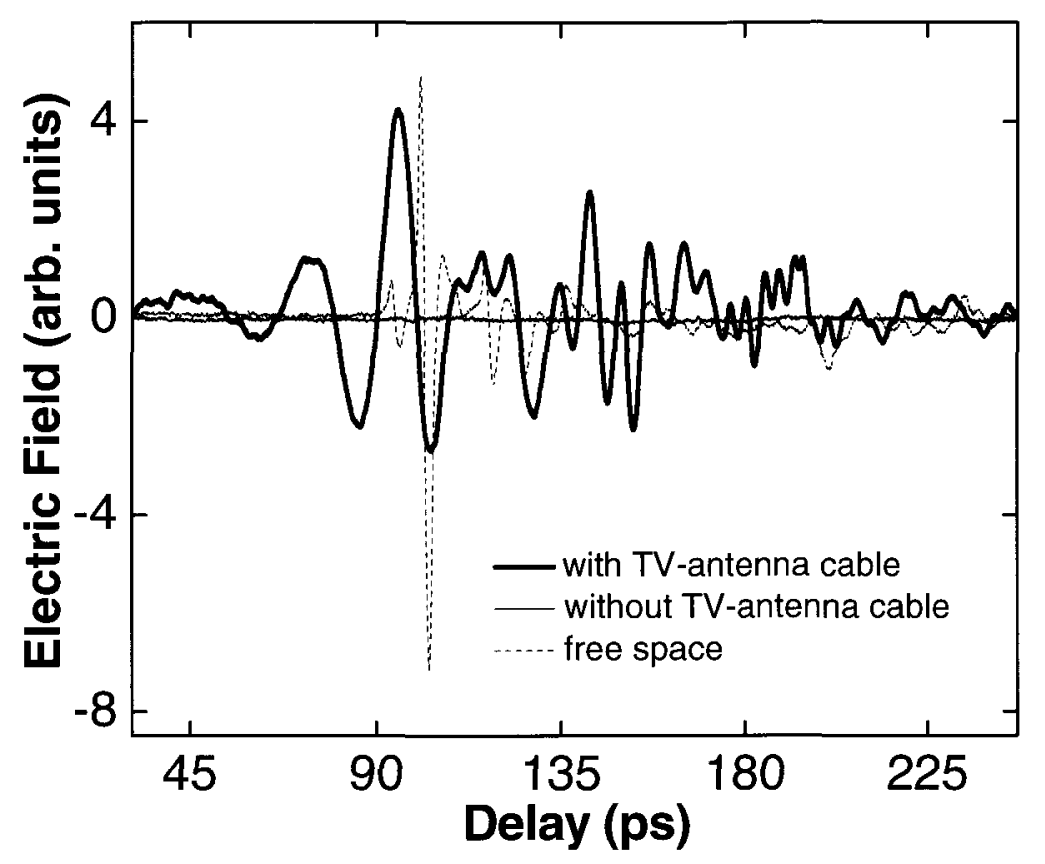

Fig. 3.2. Transmitted THz pulses. Solid: $9.5 \mathrm{~cm}$ TV-antenna cable present, with $\mathrm{THz}$ emitter and receiver at $90^{\circ}$. Thin: TV-antenna cable is absent with $\mathrm{THz}$ emitter and receiver at $90^{\circ}$. Dashed: $\mathrm{TV}$-antenna cable is absent with $\mathrm{THz}$ emitter and receiver facing each other.

The $\mathrm{THz}$ waveforms for all three measurements are shown in Fig. 3.2. For the signal with the antenna cable, we observe a positive chirp in the initial signal, where the high frequency components arrive later in time. This dispersive behavior is distinct from the usual fundamental TEM mode propagating in the two-wire waveguide as demonstrated in Fig. 2.1, and is probably caused by small air gaps between the metal wires and the dielectric medium. ${ }^{21}$ The long-lived oscillations following the main burst are probably due to the excitation of higher-order waveguide modes. As expected, without the cable in place, no energy is guided from emitter to receiver. When the receiver is re-positioned to detect the freely propagating wave, we observe an undistorted pulse. 


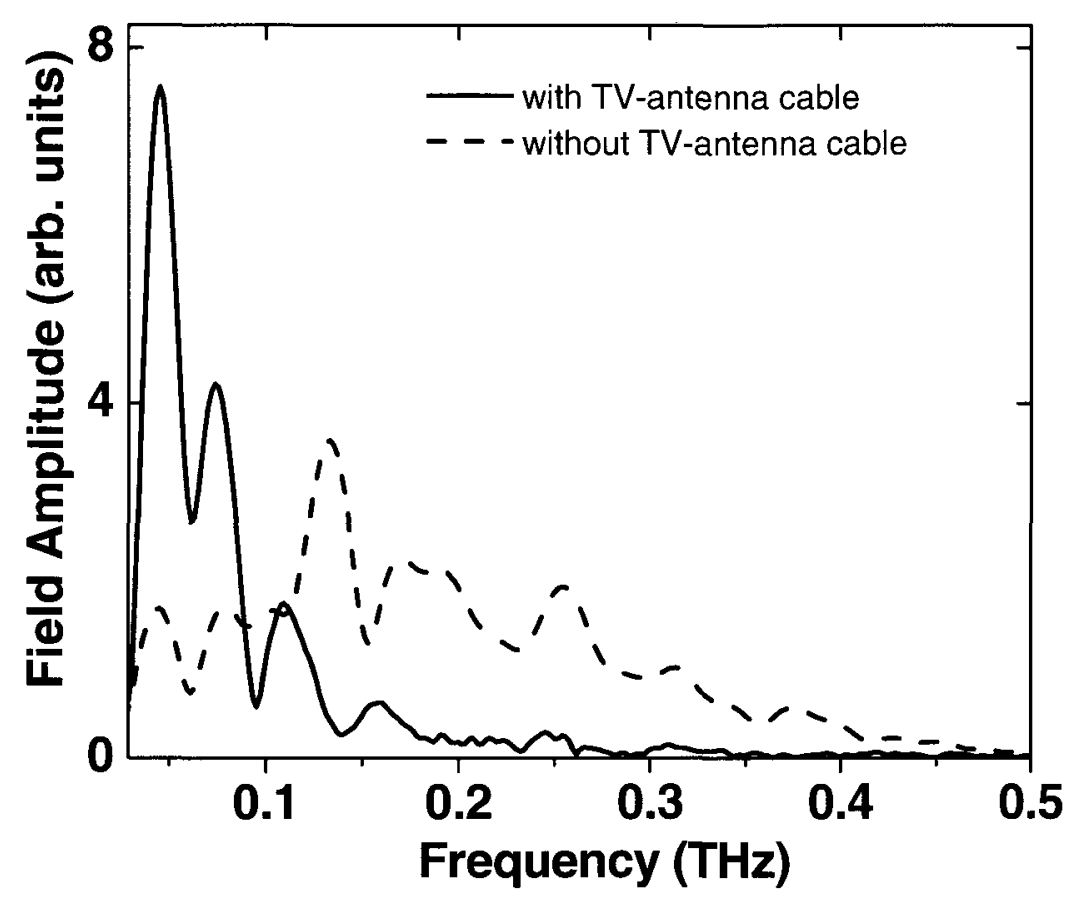

Fig. 3.3. Spectra for the $\mathrm{THz}$ waveforms. Solid: $9.5 \mathrm{~cm}$ TV-antenna cable is present with $\mathrm{THz}$ emitter and receiver at $90^{\circ}$. Dashed: TV-antenna cable is absent with $\mathrm{THz}$ emitter and receiver facing each other.

Fig. 3.3 gives the spectra corresponding to the two waveforms in Fig. 3.2. We observe greater low-frequency content in the signal with the antenna cable, compared to the free-space signal. This demonstrates that the cable has functioned as a waveguide, successfully inhibiting the diffractive losses (which are higher at low frequencies). The dramatic drop in the high-frequency content is probably due to the additional losses caused by the dielectric medium, which likely represents the dominant loss mechanism. Nonetheless, we find that this TV-antenna cable can carry frequency components up to about $0.2 \mathrm{THz}$, although these are generally designed to carry frequencies only up to about $800 \mathrm{MHz}$ (Ultra-High-Frequency (UHF) TV broadcast band). ${ }^{22}$ 


\section{CONCLUSIONS}

In summary, we have experimentally and theoretically studied the $\mathrm{THz}$ propagation characteristics of two-wire waveguides. Experimental results indicate that the mode at the end of the waveguide resemble a dipole pattern, consistent with the fundamental TEM mode of this two-conductor structure. The demonstrated low attenuation and bending loss is consistent with early low frequency (microwave) experimental and theoretical results. ${ }^{23,24} \mathrm{We}$ experimentally observe that the two-wire configuration can be used to efficiently excite the radial mode (Sommerfeld wave) of a single wire, by adiabatically separating the two wires into two single wires. We also observe that a commercial $300-\Omega$ twin-pair TV-antenna cable can be used to propagate $\mathrm{THz}$ radiation up to about $0.2 \mathrm{THz}$ at considerable bend angles. 


\section{References:}

1 G. Gallot, S. P. Jamison, R. W. McGowan, and D. Grischkowsky, J. Opt. Soc. Am. B 17, 851 (2000).

2

4

5 S. P. Jamison, R. W. McGowan, and D. Grischkowsky, Appl. Phys. Lett. 76, 1987 (2000).

R. Mendis and D. Grischkowsky, J Appl. Phys. 88, 4449 (2000).

R. Mendis and D. Grischkowsky, Opt. Lett. 26, 846 (2001).

R. Mendis and D. M. Mittleman, J. Opt. Soc. Am. B 26, A6 (2009).

R. Mendis and D. M. Mittleman, Optics Express 17, 14839 (2009).

K. Wang and D. M. Mittleman, Nature 432, 376 (2004).

T.-I. Jeon, J. Zhang, and D. Grischkowsky, Appl. Phys. Lett. 86, 161904 (2005).

K. Wang and D. M. Mittleman, J. Opt. Soc. Am. B 22 (2005).

J. A. Deibel, K. Wang, M. D. Escarra, and D. M. Mittleman, Optics Express 14, 279 (2006).

B. Bowden, J. A. Harrington, and O. Mitrofanov, Opt. Lett. 32, 2945 (2007).

W. Zhu, A. Agrawal, and A. Nahata, Opt. Express 16, 6216 (2008).

H. Han, H. Park, M. Cho, and J. Kim, Appl. Phys. Lett. 80, 2634 (2002).

M. Goto, A. Quema, H. Takahashi, S. Ono, and N. Sarukura, Jpn. J. Appl. Phys 43, 317 (2004).

M. Skorobogatiy and A. Dupuis, Appl. Phys. Lett. 90, 113514 (2007).

V. Astley, R. Mendis, and D. M. Mittleman, Appl. Phys. Lett. 95 (2009).

M. Mbonye, V. Astley, W. L. Chan, J. A. Deibel, and D. M. Mittleman, Lasers and Electro-Optics, 2007. CLEO (2007).

J. D. Jackson, JohnWiley \& Sons,NewYork, 3rd edn, 356 (1999).

R. Barrett, M. Berry, T. F. Chan, J. Demmel, J. Donato, J. Dongarra, V. Eijkhout, R. Pozo, C. Romine, and H. V. d. Vorst, SIAM (1995).

S. Ramo, J. R. Whinnery, and T. V. Duzer, John Wiley and Sons, Inc., 49 (1965).

R. Mendis, Opt. Lett. 31, 2643 (2006).

R. Schneider and J. Ross, IEEE Spectrum mag., 44 (2009).

K.Tomiyasu, Proceedings of the IRE 38, 679 (1950).

G. Goubau, Microwave Theory and Techniques, IRE Transactions 40, 1061 (1956). 